\title{
Examination of Three Case Studies of Caring for Psychiatric Patients on a Medical Surgical Unit
}

\author{
Victoria Siegel \\ Molloy College, Rockville Centre, New York, USA \\ Email: vsiegel@molloy.edu \\ Received 20 October 2014; revised 19 November 2014; accepted 30 November 2014 \\ Academic Editor: Donna M. Wilson, University of Alberta, Canada \\ Copyright (C) 2014 by author and Scientific Research Publishing Inc. \\ This work is licensed under the Creative Commons Attribution International License (CC BY). \\ http://creativecommons.org/licenses/by/4.0/ \\ (c) (i) Open Access
}

\begin{abstract}
Medical surgical nurses frequently care for patients with psychiatric disorders as one in four people have a diagnosed psychiatric disorder. Since these nurses specialize in medical surgical nursing, they often feel they lack the expertise to care for patients with co-morbid psychiatric problems. This article presents three case studies and delineates nursing care strategies which could assist the medical-surgical nurses in caring for this population.
\end{abstract}

\section{Keywords}

Psychiatric Patients, Medical Surgical Unit, Nursing Education, Case Studies

\section{Introduction}

Historically, health care professionals have been educated in silos. In recent years, inter-professional education has been addressed in the literature so the different professions understand and respect each other's roles and contributions to the health care team. In addition, we care for our patients in silos and separate psychiatric patients from medical-surgical patients. This separation leads to the caretakers being expert in their care, yet leaves the patients with dual problems without the expertise of their caregivers in both areas.

The purpose of this article is to address this issue and increase the comfort level of the medical-surgical nurse in caring for patients who also have psychiatric problems. Suffice it to say, on the flip side the psychiatric nurses would also not be comfortable assessing a post-op patient or caring for patients who have chest tubes!

Mental disorders are common in the United States. Over twenty six percent of Americans 18 or older, one in 
four adults, suffer from a diagnosable mental disorder in a given year. This translates into approximately 57.7 million people [1].

Many people do not seek psychiatric treatment due to the stigma of this category of illnesses or due to a lack of insurance coverage. Since such a high percentage of adults have mental illness, many of them also have medical or surgical needs and are admitted to medical surgical units. The health care system perpetuates the stigma of mental illness in subtle ways by not having a psychiatric patients name on the outside of the chart when all of the medical surgical patient's names are on the outside of charts. In so doing, we are "advertising" that it is something to be ashamed of.

Many nurses on medical-surgical units are sometimes uncomfortable caring for patients who also have a psychiatric disorder. I sometimes hear "I didn't go into psych for a reason" or "Psych scares me. I don't know what to say to psych patients.” Yet, frequently, patients with psychiatric diagnoses are admitted to medical-surgical units.

Let's examine a few case studies.

\section{Case Presentation 1}

A 51-year-old female with a history of bipolar disorder presented to the ER claiming she was feeling suicidal. She had jumped from a second story window and c/o pain in her back and both ankles. She was alert and oriented, but combative on admission to ED. C-collar in place, handcuffed to stretcher. "I was on the floor, my husband was trying to hurt me, so I jumped." "I wanted to hurt myself, and I wanted to hurt my husband."

The police reported that the patient was aggressive towards husband with scissors, attempted to cut self on wrists. Patient's husband has a restraining order against her. "She reported that her husband had been abusive to her through the years and he recently threatened to kill her." Her ex-husband reported she has been deteriorating for 5 weeks, belligerent, aggressive, not sleeping and drinking wine.

On admission — the patient reported she was depressed, suicidal, and scared. She reported a recent decreased need for sleep, euphoric mood, and racing thoughts. Mental status: appearance-poor hygiene, disheveled; motor activity—restless; affect—agitated; mood—depressed and anxious; speech—soft; thought process—circumstantial; judgment—poor impulse control, maladaptive; Insight—poor. Intelligence is below average. Concentration- distracted.

Pt. suffered multiple comminuted fractured of her left ankle and fracture of L5, these injuries resulted from her jumping out of the second story window. Surgical intervention was not needed but the patient was admitted to a medical surgical unit.

Psych history-she reported past history of manic symptoms along with psychotic symptoms. A decreased need for sleep, euphoria, bizarre behavior, racing thoughts, and increased goal oriented activity has led to hospitalizations in the past along with paranoia. She has had 12 psych admissions, most recent admission was 2 years ago. Reports one past suicide attempt 6 yrs. ago when she left her husband and was in a shelter. She was discovered by staff and brought to the hospital. She then divorced her husband.

She reports an occasional glass of beer or wine, no street drugs or abuse prescribed medications. However, her blood alcohol level was negative and urine toxicology screen was negative.

Family psych history — mother had schizophrenia and alcohol dependence and her niece committed suicide.

Hospital course - on admission patient was depressed and reported feelings of hopelessness, helplessness, suicidal ideation and paranoia. Patient was started on Risperadal, discontinued from Wellbutrin as it was thought it may have been activating the patient and inducing mixed symptoms as well as mania. Patient also placed on Zoloft for depressed mood and continued on Depakote. Mood improved, her affect is stabilized and her paranoia symptoms diminished. The patient remained on the medical-surgical floor for the 13 days of her hospitalization. She was managed very well on the unit, and did not have any behavioral problems. She was followed by the psych NP, nurses and her MD. The nurses reported not understanding her condition and they were wary of caring for her.

\subsection{Discussion}

In the above case presentation the nurses reported being wary of caring for the patient and not knowing what to say to her and/or how to interact with her. Many people have bipolar disorder and it would be helpful if medical-surgical nurses understood the illness and how to care for persons with the disorder. 
"Bipolar disorder is a chronic illness with recurring episodes of mania and depression that can last from one day to months. This mental illness causes unusual and dramatic shifts in mood, energy and the ability to think clearly." "More than 10 million Americans have bipolar disorder." Bipolar disorder can be managed successfully with medication [2].

By educating the medical-surgical nurses regarding bipolar disorder and therapeutic communication techniques listed below, they would have been more comfortable in caring for the above patient.

Therapeutic Communication Techniques:

1. Use of broad opening statements, which encourages the patient to take the lead in a conversation.

2. Giving recognition-indicates awareness of an accomplishment

3. Making observations—-sharing perceptions of behaviors

4. General leads-implies the nurse is following the course of the conversation and is interested in hearing more.

5. Reflecting feelings restating content-mirrors feelings and ideas the patient has expressed and directed it back to the patient.

6. Verbalizing the implied—allows nurse to validate feelings, ideas expressed.

7. Clarifying content—clarifies unclear communication and helps patient clarify own thoughts or feelings.

8. Offering self/silence - this is the most important technique and it communicates caring and is the essence of nursing.

9. Presenting reality-assures the patient of what is real to the nurse.

10. Voicing doubt-used to give patient an opportunity to re-examine negative beliefs about self or to allay fears arising from distorted perception [3].

\subsection{Conclusion}

The patient in case study \#1 was not problematic on the unit, but the nurses felt ill prepared to care for her psychiatric disorder. By increasing understanding of mental illnesses and the use of therapeutic communication techniques, nurses will feel more comfortable and patients will benefit from their care.

\section{Case Presentation 2}

31-year-old woman with a history of: depression; seizure disorder; anemia; diabetes; hypertension; end stage renal disease; polysubstance abuse and malfunction of hemodialysis catheter.

When the patient was age 14, she was diagnosed with diabetes, this led to hypertension; she had a son at age 21 and the pregnancy led to kidney failure and the need to go on dialysis. She has since had several access revisions. The patient also reported having a history of depression, anxiety and post-traumatic stress disorder (but would not elaborate).

When I first introduced myself to this patient, she was very angry and was facing away from me, staring at the wall. She was on isolation for MRSA and VRE and this truly made her feel more isolated. I asked if I could assist her with anything and she said she needed some sheets on her bed so she could lie down and she needed pain medication. She was very itchy and needed the linens washed in Ivory. I offered to go and collect the sheets from housekeeping and when I came back with the sheets, she was then engaging and no longer angry and we spoke. Through my actions, I demonstrated caring and this is extremely important to remember as nurses [4].

The patient wanted to take Ambien (a sleeping medication) at 3:00 pm. The nursing staff was incorrectly viewing this as drug seeking behavior. She did not truly want to take Ambien at $3 \mathrm{pm}$, but was trying to express how poorly she felt and she wanted the nurses to understand this.

\subsection{Discussion}

Perhaps the nursing staff would have been more comfortable caring for this patient if they understood poly-substance abuse and the frequency with which persons with mental illness self-medicate with alcohol or illegal substances. It is referred to as dual diagnosis.

"Dual diagnosis is a term used to describe people with mental illness who have coexisting problems with drugs and/or alcohol. The relationship between the two is complex, and the treatment of people with co-occurring substance abuse (or substance dependence) and mental illness is more complicated than the treatment of either condition alone. This is unfortunately a common situation—many people with mental illness have ongoing 
substance abuse problems, and many people who abuse drugs and alcohol also experience mental illness. Drugs and alcohol can be a form of self-medication. In such cases, people with mental illness may have untreated—or incompletely treated - conditions (such as anxiety or depression) that may 'feel less painful' when the person is high on drugs or alcohol. Unfortunately, while drugs and alcohol may feel good in the moment, abuse of these substances doesn't treat the underlying condition and—almost without exception—makes it worse” [5].

\subsection{Conclusions}

By educating the medical-surgical nurses about psychiatric illnesses and poly substance abuse, the nurses will feel more comfortable and confident in caring for this population. Any person who is hospitalized is in a crisis, this may be perceived much worse by a patient with mental illness who also may have had very bad experiences with hospitals and possibly has had involuntary hospital stays. Many times patients with mental illness are brought to hospitals by the police and in handcuffs. The following case study demonstrates stigma and mistrust of the system.

\section{Case Presentation 3}

78-year-old man with sudden onset of RUQ pain woke him up from sleep at 2:30 am. The pain was sharp, non-radiating, no fever, chills, $\mathrm{n} / \mathrm{v}$. An abdominal ultrasound showed a fatty liver, gall stones and gall bladder dilation. The relevant lab results were: AST-555; ALT-482.

His past health history includes the following: gout; high cholesterol; schizophrenia; hyperlipidemia; type 2 diabetes; GERD and previous major depressive breakdowns which required prolonged psychiatric hospitalizations.

The admitting diagnosis was acute cholecystitis, cholelithiasis. The medications he took at home are: Prilosec; Haldol; Depakote; Lipitor; metformin. After medical clearance, he will have laparoscopic cholecystectomy.

The patient was very angry that his wife told the staff that he had a psychiatric problem, because he thinks mental illness has a stigma. When the nurse offered his nighttime Haldol medication, he refused and said "I don't take that!" So, he refused his medication in a hospital because he was embarrassed and felt that mental illness was a stigma. He didn't trust the system, he didn't trust the nurses.

\subsection{Discussion}

What does this say about our society and mental illness? Perhaps, as nurses we can do a better job to demonstrate compassion, caring and educate the community to assist in breaking down the barriers to health care and de-stigmatizing mental illness. Patients and their families have an easier time disclosing medical problems than psychiatric problems to others, including medical professionals. The above patient experienced internalized stigma.

"Internalized stigma, also referred to as self or felt stigma, exists at the individual (i.e., micro) level and, in the context of mental illness can be described as a process whereby affected individuals endorse stereotypes about mental illness, anticipate social rejection, consider stereotypes to be self-relevant, and believe they are devalued members of society [6].

\section{Medication and Counseling Education}

- Support adherence to medication.

- Medication non-adherence is a primary factor in relapse and re-hospitalization.

- Ask patient for input on the medications that have been helpful in the past, serve as a pt. advocate [3].

- Counseling interventions support patients' ability to develop effective coping strategies

- Counseling interventions by psych NP or psychiatrist and reinforced by medical surgical nurse can be very helpful, this can decrease anxiety and behavioral problems can be averted.

\subsection{Conclusion}

The pervasive problem of the stigma of mental illness is also evident in the case of a 60 -year-old woman admitted for a suicide attempt by over-dosing on pills, history of depression and on a 1:1. She did not want to be admitted to psych because psych has a stigma and didn't want her friends and neighbors to know that she was in a psych unit. 
The medical surgical nurses need to be aware of the safety issues for their patients, particularly those admitted post suicide attempt or with depression. The Joint commission reported 827 inpatient suicides in its sentinel events report in November of 2010. The report outlined the following: 14.25\% occurred in non-behavioral health units of general hospitals (medical-surgical units, ICU, oncology, telemetry); $8.02 \%$ in the ED; $2.45 \%-$ home care, long term care, and physical rehabilitation. The locations of these incidences included the bathroom, closet, and showers. Of those who attempt suicide, $62.3 \%(678,000)$ received medical attention and $46 \%$ (500, 000) stayed overnight or longer for their suicide attempts [7].

Patients who have attempted suicide are more safely cared for on a psychiatric unit as they are designed with safety and structure in mind.

\section{Summary}

In summary, medical surgical nurses frequently care for patients with psychiatric problems and if they are more aware of the various illnesses, medications, stigma of mental illness, they will have increased comfort when caring for this patient population using therapeutic communication techniques, giving of self, using silence and preventing escalation of anger and agitation. As in the case studies we examined, many psychiatric patients on medical surgical units did not lose control and were properly medicated for their psychiatric problems.

\section{References}

[1] National Institute of Mental Health (NIMH) The Numbers Count: Mental Disorders in America. http://www.nimh.nih.gov/health/statistics/index.shtml

[2] National Alliance of Mental Illness (NAMI) Bipolar Disorder. http://www.nami.org/factsheets/bipolardisorder_factsheet.pdf

[3] Videbeck, S. (2010) Psychiatric Mental Health Nursing. 4th Edition, Lippincott Williams and Wilkins, Philadelphia.

[4] Watson, J. (2008) Nursing, the Philosophy and Science of Caring. Revised Edition, University Press of Colorado, Boulder.

[5] National Alliance of Mental Illness (NAMI). http://www.nami.org/template.cfm?template=/contentManagement/contentDisplay.cfm\&contentID=23049

[6] Livingston, J. and Boyd, J. (2010) Correlates and Consequences of Internalized Stigma for People Living with Mental Illness: A Systematic Review and Meta-Analysis. Social Science and Medicine, 71, 2150-2161. http://dx.doi.org/10.1016/j.socscimed.2010.09.030

[7] Joint Commission (2010) Sentinel Event Alert, Issue 46. http://www.jointcommission.org/sentinel_event_alert_issue_46_a_follow-up_report_on_preventing_suicide_focus_on _medicalsurgical_units_and_the_emergency_department/ 
Scientific Research Publishing (SCIRP) is one of the largest Open Access journal publishers. It is currently publishing more than 200 open access, online, peer-reviewed journals covering a wide range of academic disciplines. SCIRP serves the worldwide academic communities and contributes to the progress and application of science with its publication.

Other selected journals from SCIRP are listed as below. Submit your manuscript to us via either submit@scirp.org or Online Submission Portal.
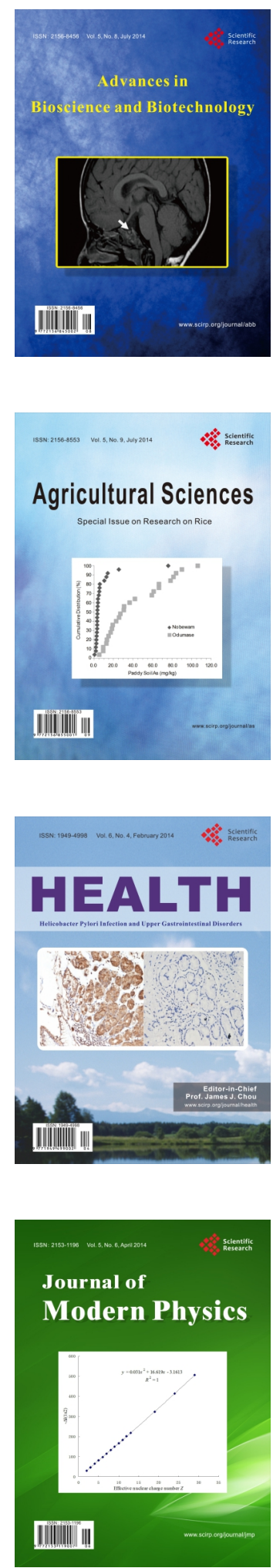
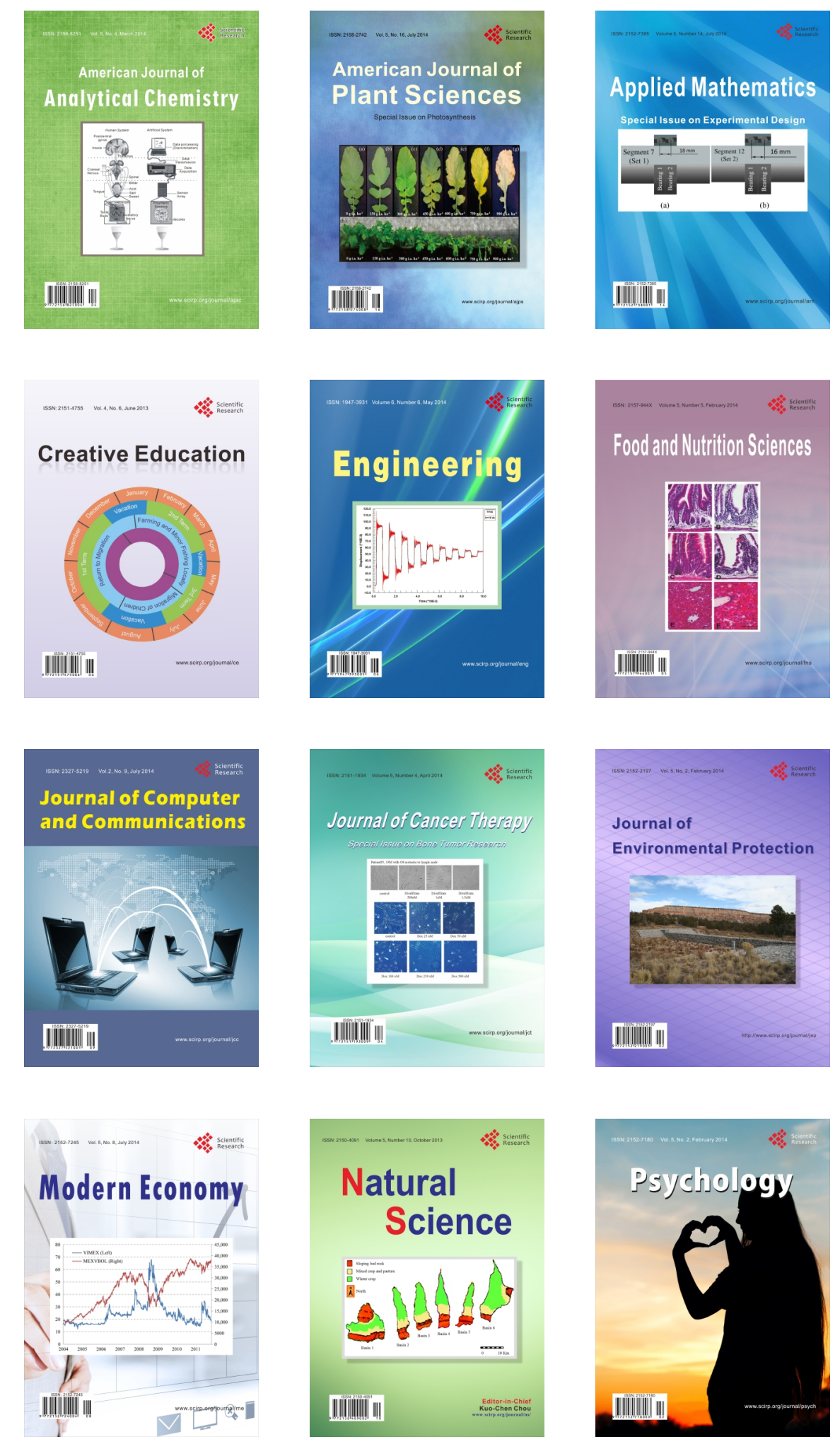